\title{
Spacecraft motion around artificial equilibrium points
}

\author{
A. K. de Almeida Jr.® - A. F. B. A. Prado • \\ T. Yokoyama - D. M. Sanchez
}

Received: 27 March 2017 / Accepted: 19 November 2017 / Published online: 5 December 2017

(C) Springer Science+Business Media B.V., part of Springer Nature 2017

\begin{abstract}
The main goal of this paper is to describe the motion of a spacecraft around an artificial equilibrium point in the circular restricted three-body problem. The spacecraft is under the gravitational influence of the Sun and the Earth, as primary and secondary bodies, subjected to the force due to the solar radiation pressure and some extra perturbations. Analytical solutions for the equations of motion of the spacecraft are found using several methods and for different extra perturbations. These solutions are strictly valid at the artificial equilibrium point, but they are used as approximations to describe the motion around this artificial equilibrium point. As an application of the method, the perturbation due to the gravitational influence of Jupiter and Venus is added to a spacecraft located at a chosen artificial equilibrium point, near the $L_{3}$ Lagrangian point of the Sun-Earth system. The system is propagated starting from this point using analytical and numerical solu-
\end{abstract}

\footnotetext{
A. K. de Almeida Jr. (凶) • A. F. B. A. Prado .

D. M. Sanchez

Instituto Nacional de Pesquisas Espaciais (INPE),

São José dos Campos, SP, Brazil

e-mail: allan.junior@inpe.br

A. F. B. A. Prado

e-mail: antonio.prado@inpe.br

D. M. Sanchez

e-mail: diogo.sanchez@inpe.br

T. Yokoyama

Universidade Estadual Paulista (UNESP), Rio Claro, SP,

Brazil

e-mail: tadashi@rc.unesp.br
}

tions. Comparisons between analytical-analytical and analytical-numerical solutions for several kinds of perturbations are made to guide the choice of the best analytical solution, with the best accuracy.

Keywords Astrodynamics · Equilibrium points · Restricted three-body problem · Nonlinear systems

\section{Introduction}

The Lagrangian equilibrium points that appear in the restricted three-body problem are convenient for astronautical applications. They are good candidates to place spacecraft, since their equilibrium conditions help to reduce the fuel consumption for station-keeping maneuvers. An interesting application is the use of one of these points $\left(L_{3}\right)$ in the Sun-Earth system to place a spacecraft. Such spacecraft could help to observe and detect solar activities in the momentary opposite side of the Sun from the perspective of the Earth. Combined with the rotation of the Sun, these detections could improve the prediction of coronal mass ejection in the direction of the Earth weeks in advance. This advantageous point can be useful for many others spacecraft missions, such as collections of particles traveling in space [1] or for parallax measurements. Even considering these benefits, there are only a few investigations performed to explore this point. One of the reasons is its instability. Another important problem is the strong perturbations coming from other planets, in particular 
Jupiter and Venus [2]. This is specially true during the times that they are closer to this point. The instability problem exists not only for the point itself, but also for the motion around this point [3]. This instability is also present in the other two collinear equilibrium points, called $L_{1}$ and $L_{2}$, but many real applications are considered for these two points [4-8]. It is necessary to study options to control this instability, but it can be done using an adequate station-keeping strategy. Among the few studies related to this particular point, it can be mentioned in reference [9], which considers motions around these points, and researches studying transfer orbits $[10,11]$.

Moreover, equilibrium points are not restricted to the Lagrangian points. If some extra force is taken into account, then families of equilibrium points can arise $[12,13]$. These points are the so-called artificial equilibrium points (AEP), which are points of equilibrium obtained under a more complex dynamics, which has the addition of more forces besides the gravity of the bodies involved. These extra forces can shift the location of the equilibrium point, but it can also play a role in the stability of the point [14]. These AEP can be found through a concept using reflectors to generate thrust without the use of propellant, which origins goes back to 1920 s $[15,16]$. An interesting idea was patented and published by Forward in 1990s [17], where an application of the AEP allows a "statite" spacecraft to observe regions near the poles of the Earth. Since the idea of Forward, several researches have been done to investigate the AEP in the circular restricted three-body problem [13] and in the elliptic restricted three-body problem [18]. Stability of the AEP is also considered in the literature [19], including the stability of the AEP with the primary body assumed to be an oblate spheroid [20]. Changing the equilibrium point $L_{3}$ and turning it into an AEP in a new position near $L_{3}$ can be suitable for many missions, since it can offer much more options to choose a place for a "stationary" spacecraft or to place a spacecraft that orbits this point. An application of the idea of an extra force due to the solar radiation pressure to shift the location of an equilibrium point is mentioned in [21], to find places to locate a solar reflector around the Earth to redirect solar rays to slightly increase the global temperature of the Earth. A similar idea is already considered in [22], but to decrease the global temperature of the Earth. Low-thrust systems applied to AEP are studied in [23]. Attitude and trajectory stability of solar sails have been recently stud- ied in [24]. In order to generate thrust in a spacecraft without the use of propellant, solar radiation pressure is not the only option. Momentum flux of the solar wind can also be used through the so-called electric sail $[25,26]$.

In this context, the present paper has the goal of searching for solutions for the motion of a spacecraft near an artificial equilibrium point. It is assumed that the spacecraft is subjected to the force coming from the solar radiation pressure, besides the forces given by the circular restricted three-body problem Sun-Earthspacecraft. It is also considered that the spacecraft is equipped with a solar sail. An adequate choice of the parameters involved in the solar sail, like its attitude, reflectance properties, area, can generate families of locations for these new artificial equilibrium points described before, with some of them lying even outside the orbital plane of the Earth. Of course, there is an extra engineering work in building solar sails with large ratio area to mass, but they can also be used in scientific experiments, like producing energy or collecting particles traveling in space [1]. Thus, a perturbation is added to the problem through its equations of motion; then, analytical solutions of these equations are found. Analytical solutions for the equations of motion are found for some different forms of perturbations and through the use of different methods, either direct analytical solution or the analytical solution of the linearized equations of motion. From these analytical solutions, it is possible to study the motion of the spacecraft in some detail. The method is applied considering the important perturbations coming from Jupiter and Venus or Jupiter only gravitational interactions with the spacecraft located near the $L_{3}$ Lagrangian point of the Sun-Earth system. These two planets represent the largest forces acting as perturbations over a spacecraft in this location. Hence, specific values of the parameters and perturbations are given. Results coming from the different analytical methods are shown, as well as coming from a numerical method. Thus, the results are compared with each other. These comparisons guide the choice of the best model to be used, as a function of the accuracy desired for the problem and the time span involved.

This article is divided as follows. In Sect. 2, mathematical models are presented. In Sects. 2.1, 2.2 and 2.3, solutions are found through different methods and different forms for the perturbation. In Sect. 3, results are obtained for some specific cases shown in 
Sects. 3.1, 3.2, 3.3 and 3.4. Finally, Sect. 4 shows a conclusion of the paper.

\section{Mathematical models}

Suppose a spacecraft under the gravitational influence of the Sun and the Earth, subjected to a force due to the solar radiation pressure over its sail, and to perturbations from other sources. In a non-inertial rotating frame of reference, that has the Sun fixed in its center and the Earth fixed along the $x$ axis, according to the Coriolis theorem [27], the equation of motion of such spacecraft is given by

$$
\begin{aligned}
& \frac{\mathrm{d}^{2} \mathbf{r}_{s}}{\mathrm{~d} t^{2}}+2 \omega \times \frac{\mathrm{d} \mathbf{r}_{s}}{\mathrm{~d} t}+\omega \times\left(\omega \times \mathbf{r}_{s}\right)+\frac{\mathrm{d} \boldsymbol{\omega}}{\mathrm{d} t} \times \mathbf{r}_{s} \\
& =-\frac{\mu_{s}}{r_{s}^{3}} \mathbf{r}_{s}-\frac{\mu_{e}}{r_{e}^{3}} \mathbf{r}_{e}+\frac{1}{m} \mathbf{f}_{p}+\mathbf{a},
\end{aligned}
$$

where $\omega$ is the angular velocity of the rotating frame; $\mathbf{r}_{s}$ is the position of the spacecraft; $\mathbf{r}_{e}$ locates the spacecraft with respect to the Earth; $\mathbf{f}_{p}$ is the force over the solar sail due to the solar radiation pressure; $\mathbf{a}$ is the perturbative acceleration vector acting in the spacecraft; $\mu_{s}$ is the gravitational parameter of the Sun; $\mu_{e}$ is the gravitational parameter of the Earth.

The rotating frame of reference and the geometry of the problem are shown in Fig. 1, from where it is possible to see the bodies involved (Sun, Earth and spacecraft). The Sun is placed in the center of the reference system, not in the baricenter. For the purpose of this work, the motion of the Earth around the Sun is circular and non-perturbed by any force, which indicates

$\omega=(0,0, \omega)$,

where $\omega$ is a constant.
The equation of motion [Eq. (1)] now becomes

$$
\begin{gathered}
\frac{\mathrm{d}^{2} \mathbf{r}_{s}}{\mathrm{~d} t^{2}}+2 \omega \times \frac{\mathrm{d} \mathbf{r}_{s}}{\mathrm{~d} t}+\omega \times\left(\omega \times \mathbf{r}_{s}\right) \\
=-\frac{\mu_{s}}{r_{s}^{3}} \mathbf{r}_{s}-\frac{\mu_{e}}{r_{e}^{3}} \mathbf{r}_{e}+\frac{1}{m} \mathbf{f}_{p}+\mathbf{a} .
\end{gathered}
$$

The artificial equilibrium point (AEP) is defined by the condition given by

$\frac{1}{m} \mathbf{f}_{p}=\omega \times\left(\omega \times \mathbf{r}_{s}\right)+\frac{\mu_{s}}{r_{s}^{3}} \mathbf{r}_{s}+\frac{\mu_{e}}{r_{e}^{3}} \mathbf{r}_{e}$.

The reason why this condition can be called an AEP will be explained later in this section. If Eq. (4) is true, then Eq. (3) becomes:

$\frac{\mathrm{d}^{2} \mathbf{r}_{s}}{\mathrm{~d} t^{2}}+2 \boldsymbol{\omega} \times \frac{\mathrm{d} \mathbf{r}_{s}}{\mathrm{~d} t}=\mathbf{a}$

Using Eq. (2), the components of Eq. (5) can be written as

$\frac{\mathrm{d}^{2} x}{\mathrm{~d} t^{2}}-2 \frac{\mathrm{d} y}{\mathrm{~d} t} \omega-a_{x}(x, y, z, t)=0$,

$\frac{\mathrm{d}^{2} y}{\mathrm{~d} t^{2}}+2 \frac{\mathrm{d} x}{\mathrm{~d} t} \omega-a_{y}(x, y, z, t)=0$,

and

$\frac{\mathrm{d}^{2} z}{\mathrm{~d} t^{2}}-a_{z}(x, y, z, t)=0$,

where $x, y$ and $z$ are the components of the position vector $\mathbf{r}_{s}$ and $a_{x}(x, y, z, t), a_{y}(x, y, z, t)$ and $a_{z}(x, y, z, t)$ are the components of the perturbation vector $\mathbf{a}$ and may depend on the position and time variables.

Equations (6)-(7) form a set of coupled ordinary differential equations. Depending on the form of the perturbation vector a, analytical solutions for these equations may be easily obtained. In the case where the components of the perturbation vector $\mathbf{a}=\left(a_{x}, a_{y}, a_{z}\right)$ are given by
Fig. 1 Geometry of the problem in the rotating frame of reference

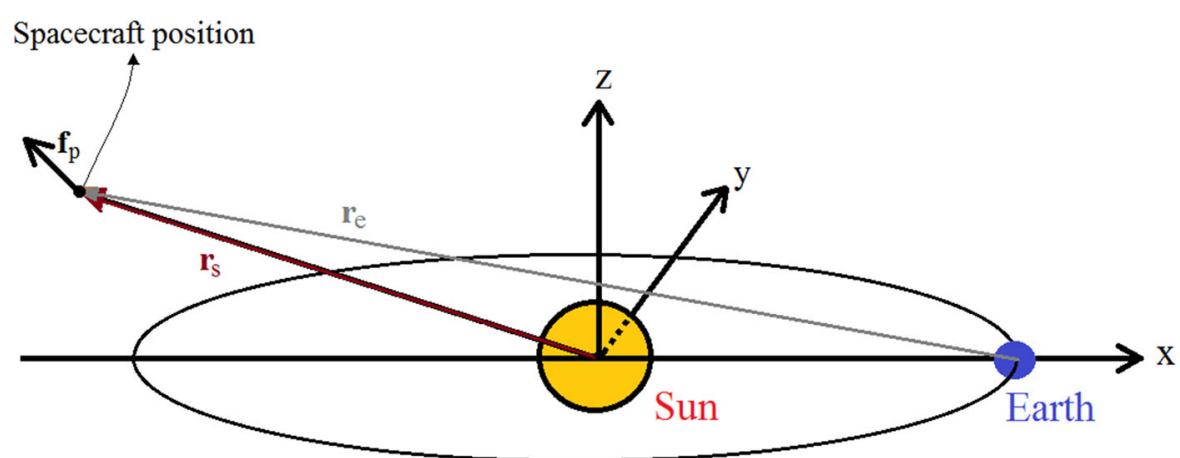


$a_{x}=\frac{1}{2 \omega} \frac{\mathrm{d} X(x)}{\mathrm{d} t}+Y(y)+T_{x}(t)$

$a_{y}=X(x)-\frac{1}{2 \omega} \frac{\mathrm{d} Y(y)}{\mathrm{d} t}+T_{y}(t)$

and

$a_{z}=Z(z)+T_{z}(t)$

where $X(x), Y(y)$ and $Z(z)$ are any functions of $x, y$ and $z$ only, respectively, and $T_{x}(t), T_{y}(t)$ and $T_{z}(t)$ are any explicit functions of time only, then Eqs. (6)-(7) can be rewritten as

$\frac{\mathrm{d}^{3} x}{\mathrm{~d} t^{3}}+4 \omega^{2} \frac{\mathrm{d} x}{\mathrm{~d} t}-2 \omega X-2 \omega T_{y}-\frac{1}{2 \omega} \frac{\mathrm{d}^{2} X}{\mathrm{~d} t^{2}}$

$-\frac{\mathrm{d} T_{x}}{\mathrm{~d} t}=0$,

$\frac{\mathrm{d}^{3} y}{\mathrm{~d} t^{3}}+4 \omega^{2} \frac{\mathrm{d} y}{\mathrm{~d} t}+2 \omega Y+2 \omega T_{x}+\frac{1}{2 \omega} \frac{\mathrm{d}^{2} Y}{\mathrm{~d} t^{2}}$

$-\frac{\mathrm{d} T_{y}}{\mathrm{~d} t}=0$,

and

$\frac{\mathrm{d}^{2} z}{\mathrm{~d} t^{2}}-Z-T_{z}=0$

Equations (12)-(14) form a set of three uncoupled ordinary differential equations. Depending on the form of $X, Y, Z, T_{x}, T_{y}$, and $T_{z}$, analytical solutions for these equations may be obtained.

Supposing a perturbation caused by the gravitational interaction of the spacecraft with another planet, it depends on the relative position of the spacecraft with respect to the planet, which means that it depends only on the position of the spacecraft and the time $t$, since the position of the perturbing planet is usually supposed to be known as a function of time. The major variation in the relative position between the spacecraft and the perturbing planet is due to the variation in the position of the planet itself in the frame of reference, because, in comparison, the displacement of the spacecraft in this same frame of reference is supposed to be small around the AEP. Therefore, it is assumed here the approximation that the perturbing force may be calculated using the relative position between the planet and the AEP. Note that in this approximation, the advantage that the perturbing force becomes only a function of time $t$ is used in the next solution.

\subsection{Analytical solution 1: perturbation linearly dependent on time}

If the gravitational forces (or accelerations) due to Venus and Jupiter are used as an example of perturbation, then this perturbation is a function of the position of the spacecraft and time, because the motion of Venus and Jupiter around the Sun can be predicted as a function of time. The motion is close around the AEP; then, the perturbation can be approximated by a function of time only, calculated at the initial position of the spacecraft. More than that, the components of the perturbation are assumed to be linear functions of time in the following forms:

$$
\begin{aligned}
a_{x}(x, y, z, t)= & a_{x}\left(x_{0}, y_{0}, z_{0}, t\right)=a_{x}\left(x_{0}, y_{0}, z_{0}, 0\right) \\
& +\alpha_{x} t, \\
a_{y}(x, y, z, t)= & a_{y}\left(x_{0}, y_{0}, z_{0}, t\right)=a_{y}\left(x_{0}, y_{0}, z_{0}, 0\right) \\
& +\alpha_{y} t,
\end{aligned}
$$

and

$a_{z}(x, y, z, t)=a_{z}\left(x_{0}, y_{0}, z_{0}, t\right)=a_{z}\left(x_{0}, y_{0}, z_{0}, 0\right)$

$$
+\alpha_{z} t
$$

where $x_{0}, y_{0}$ and $z_{0}$ are the initial position components and $\alpha_{x}, \alpha_{y}$ and $\alpha_{z}$ are assumed to be constants calculated as explained now. For a given time $t=t_{f}>0$, the value of the constant $\alpha_{x}$ is calculated using Eq. (15) itself, because the perturbation $a_{x}\left(x_{0}, y_{0}, z_{0}, t_{f}\right)$ at the time $t=t_{f}$ and the perturbation $a_{x}\left(x_{0}, y_{0}, z_{0}, 0\right)$ at the time $t=0$ are assumed to be known. In the same way, Eqs. (16) and (17) can be used to calculate the values of $\alpha_{y}$ and $\alpha_{z}$, respectively. For brevity and simplicity, instead of using the notation given by Eqs. (15-17), the perturbation components are written by following the notation given by

$a_{x}=a_{x 0}+\alpha_{x} t$,

$a_{y}=a_{y 0}+\alpha_{y} t$,

and

$a_{z}=a_{z 0}+\alpha_{z} t$

where $a_{x 0}=a_{x}\left(x_{0}, y_{0}, z_{0}, 0\right)$ and $\alpha_{x}=\frac{1}{t_{f}}\left[a_{x}\left(x_{0}, y_{0}\right.\right.$, $\left.\left.z_{0}, t_{f}\right)-a_{x}\left(x_{0}, y_{0}, z_{0}, 0\right)\right]$ in Eq. (18) and analogously for Eqs. (19) and (19).

If the relations $X(x)=0 ; Y(y)=0 ; Z(z)=0$; $T_{x}(t)=a_{x 0}+\alpha_{x} t ; T_{y}(t)=a_{y 0}+\alpha_{y} t$; and $T_{z}(t)=$ $a_{z 0}+\alpha_{z} t$ are put into Eqs. (9)-(11), the components of the perturbation given by Eqs. (18)-(19) are satisfied. 
Therefore, using these relations, uncoupled Eqs. (12)(14) becomes

$\frac{\mathrm{d}^{3} x}{\mathrm{~d} t^{3}}+4 \omega^{2} \frac{\mathrm{d} x}{\mathrm{~d} t}-2 \omega T_{y}-\frac{\mathrm{d} T_{x}}{\mathrm{~d} t}=0$,
$\frac{\mathrm{d}^{3} y}{\mathrm{~d} t^{3}}+4 \omega^{2} \frac{\mathrm{d} y}{\mathrm{~d} t}+2 \omega T_{x}-\frac{\mathrm{d} T_{y}}{\mathrm{~d} t}=0$,

and

$\frac{\mathrm{d}^{2} z}{\mathrm{~d} t^{2}}-T_{z}=0$

Equations (21)-(22) form a set of uncoupled ordinary differential equations. The analytical solution for these equations can be easily obtained, and they are written as functions of the initial positions and velocities as

$$
\begin{aligned}
x(t)= & \frac{\sin (2 t \omega)\left(-\frac{a_{y 0}}{2 \omega}+v_{x 0}-\frac{\alpha_{x}}{4 \omega^{2}}\right)}{2 \omega} \\
& +\frac{\cos (2 t \omega)\left(-\frac{a_{x 0}}{2 \omega}-v_{y 0}+\frac{\alpha_{y}}{4 \omega^{2}}\right)}{2 \omega} \\
& +t\left(\frac{a_{y 0}}{2 \omega}+\frac{\alpha_{x}}{4 \omega^{2}}\right)+\frac{a_{x 0}}{4 \omega^{2}}+\frac{t^{2} \alpha_{y}}{4 \omega}+\frac{v_{y 0}}{2 \omega} \\
& +x_{0}-\frac{\alpha_{y}}{8 \omega^{3}}, \\
y(t)= & \frac{\cos (2 t \omega)\left(-\frac{a_{y 0}}{2 \omega}+v_{x 0}-\frac{\alpha_{x}}{4 \omega^{2}}\right)}{2 \omega} \\
& +\frac{\sin (2 t \omega)\left(\frac{a_{x 0}}{2 \omega}+v_{y 0}-\frac{\alpha_{y}}{4 \omega^{2}}\right)}{2 \omega} \\
& -\frac{v_{x 0}\left(\frac{\alpha_{y}}{4 \omega^{2}}-\frac{a_{x 0}}{2 \omega}\right)+\frac{\alpha_{y}}{4 \omega_{0}}-\frac{t^{2} \alpha_{x}}{4 \omega}+y_{0},}{4 \omega^{2}}
\end{aligned}
$$

and

$z(t)=\frac{t^{2} a_{z 0}}{2}+\frac{t^{3} \alpha_{z}}{6}+t v_{z 0}+z_{0}$.

where $\left(x_{0}, y_{0}, z_{0}\right)=(x(0), y(0), z(0))$ and $\left(v_{x 0}, v_{y 0}, v_{z 0}\right)$ $=\left(v_{x}(0), v_{y}(0), v_{z}(0)\right)$ are the position and velocity initial conditions of the motion.
The derivatives with respect to time are the respective velocity components, and they are written as

$$
\begin{aligned}
v_{x}(t)=\cos & (2 t \omega)\left(-\frac{a_{y 0}}{2 \omega}+v_{x 0}-\frac{\alpha_{x}}{4 \omega^{2}}\right) \\
& -\sin (2 t \omega)\left(-\frac{a_{x 0}}{2 \omega}-v_{y 0}+\frac{\alpha_{y}}{4 \omega^{2}}\right)+\frac{a_{y 0}}{2 \omega} \\
& +\frac{t \alpha_{y}}{2 \omega}+\frac{\alpha_{x}}{4 \omega^{2}}, \\
v_{y}(t)=- & \sin (2 t \omega)\left(-\frac{a_{y 0}}{2 \omega}+v_{x 0}-\frac{\alpha_{x}}{4 \omega^{2}}\right) \\
& +\cos (2 t \omega)\left(\frac{a_{x 0}}{2 \omega}+v_{y 0}-\frac{\alpha_{y}}{4 \omega^{2}}\right)-\frac{a_{x 0}}{2 \omega} \\
& -\frac{t \alpha_{x}}{2 \omega}+\frac{\alpha_{y}}{4 \omega^{2}},
\end{aligned}
$$

and

$v_{z}(t)=t a_{z 0}+\frac{t^{2} \alpha_{z}}{2}+v_{z 0}$

\subsection{Analytical solution 2: constant perturbation}

In a simpler and more direct case, the perturbation a is a constant vector, calculated at the AEP in the time $t=0$, being $a_{x}(x, y, z, t)=a_{x 0}, a_{y}(x, y, z, t)=a_{y 0}$ and $a_{z}(x, y, z, t)=a_{z 0}$. This is a particular case of the situation shown in Sect. 2.1, where $\alpha_{x}=\alpha_{y}=\alpha_{z}=0$. Therefore, using these relations, the solutions given by Eqs. (24)-(29) become

$$
\begin{aligned}
x(t)= & \frac{1}{2 \omega}\left(-\left(v_{y 0}+\frac{a_{x 0}}{2 \omega}\right) \cos (2 \omega t)\right. \\
& +\left(v_{x 0}-\frac{a_{y 0}}{2 \omega}\right) \sin (2 \omega t) \\
& \left.+a_{y 0} t+\left(v_{y 0}+\frac{a_{x 0}}{2 \omega}\right)+2 \omega x_{0}\right), \\
y(t)= & \frac{1}{2 \omega}\left(\left(v_{x 0}-\frac{a_{y 0}}{2 \omega}\right) \cos (2 \omega t)\right. \\
& +\left(v_{y 0}+\frac{a_{x 0}}{2 \omega}\right) \sin (2 \omega t) \\
& \left.-a_{x 0} t-\left(v_{x 0}-\frac{a_{y 0}}{2 \omega}\right)+2 \omega y_{0}\right), \\
z(t)= & \frac{a_{z 0} t^{2}}{2}+v_{z 0} t+z_{0}, \\
v_{x}(t)= & \left(v_{y 0}+\frac{a_{x 0}}{2 \omega}\right) \sin (2 \omega t) \\
& +\left(v_{x 0}-\frac{a_{y 0}}{2 \omega}\right) \cos (2 \omega t)+\frac{a_{y 0}}{2 \omega},
\end{aligned}
$$

$v_{y}(t)=-\left(v_{x 0}-\frac{a_{y 0}}{2 \omega}\right) \sin (2 \omega t)$

$$
+\left(v_{y 0}+\frac{a_{x 0}}{2 \omega}\right) \cos (2 \omega t)-\frac{a_{x 0}}{2 \omega},
$$


and

$v_{z}(t)=a_{z 0} t+v_{z 0}$.

Now, an explanation is given for the reason why the condition given by Eq. (4) defines an AEP. In a scenario with no perturbation $\left(a_{x 0}=a_{y 0}=a_{z 0}=0\right)$, supposing that the initial velocity is given by $\left(v_{x 0}, v_{y 0}, v_{z 0}\right)=$ $(0,0,0)$, then, if Eq. (4) is true, the solutions given by Eqs. (30-35) are rewritten as

$x(t)=x_{0}$

$y(t)=y_{0}$

$z(t)=z_{0}$

$v_{x}(t)=0$

$v_{y}(t)=0$

$v_{z}(t)=0$

Equations (36)-(41) show that if there is no perturbation, the initial velocity is zero and $\mathbf{f}_{p}$ is adjusted such that Eq. (4) is satisfied at least in a single point $\left(X_{0}, Y_{0}, Z_{0}\right)$, then all the components of the accelerations are balanced and the spacecraft would be in a stationary condition at the AEP. Despite the name, it is important to note that an AEP is not only a point in the 3D space, because it also requires other variables (or parameters) implicit in the force function $\mathbf{f}_{p}$ to assume values such that Eq. (4) is satisfied. In this sense, an AEP is a set of conditions.

A more direct engineering application is found if the force $\mathbf{f}_{p}$ due to the solar radiation pressure over the solar sail can be controlled such that Eq. (4) is true along all the path. Therefore, the solutions given by Eqs. (24)-(29) or (30-35) can describe the motion for a linearly time dependent or a constant perturbation, respectively. But, considering an engineering problem, it is common that the force $\mathbf{f}_{p}$ cannot be controlled during all the time. It means that Eq. (4) cannot be true along all the path and the solution given by Eqs. (24)(29) or (30)-(35) depends on this condition to be valid. However, suppose that Eq. (4) is satisfied for a single point, called AEP. If this AEP is the starting point of the motion of a spacecraft, then it is expected that, even if this condition is not satisfied outside the AEP, Eq. (5) can describe the motion of the spacecraft around the AEP with some degree of accuracy. The closer the spacecraft is from the AEP, the higher the accuracy.

A similar approximation analysis can be made for the perturbation. Equations (24)-(29) or (30)-(35) are valid as solutions of Eqs. (6-7) only if the perturbation is linearly dependent on the time or is a constant vector. Even if this is not true, with the motion starting at the AEP, it is expected that the analytical solution given by Eqs. (24)-(29) or (30)-(35) can describe the motion originated by Eqs. (6-7) if the perturbation is approximated accordingly, being constant or linearly dependent on time.

\subsection{Linear analytical solution}

Analytical solutions for Eqs. (6-7) strictly valid around the AEP can also be obtained using a well-known method presented in this subsection [28]. The simpler case of a constant perturbation is used, but this method could also be used to search for solutions in the case of other forms of perturbations. On the other side, as will be shown later, the method presented in this subsection is highly expensive. It requires the evaluation of the eigenvectors of a $6 \times 6$ matrix and other expensive steps in order to find the final solution. Despite the costs, the solution obtained through the use of this method will be useful in order to be compared with and to validate the more direct analytical solutions previously presented. According to Eq. (3), the equation of motion with no perturbation is

$$
\begin{aligned}
& \frac{\mathrm{d}^{2} \mathbf{r}_{s}}{\mathrm{~d} t^{2}}=- 2 \omega \times \frac{\mathrm{d} \mathbf{r}_{s}}{\mathrm{~d} t}-\omega \times\left(\omega \times \mathbf{r}_{s}\right) \\
&-\frac{\mu_{s}}{r_{s}^{3}} \mathbf{r}_{s}-\frac{\mu_{e}}{r_{e}^{3}} \mathbf{r}_{e}+\frac{1}{m} \mathbf{f}_{p} .
\end{aligned}
$$

If the velocity components are defined as new variables, this system of three second-order ordinary differential equations can be converted in a system of six first-order ordinary differential equations as

$\frac{\mathrm{d} \mathbf{X}}{\mathrm{d} t}=\mathbf{F}(\mathbf{X})$,

where $\mathbf{X}$ is the vector of the variables and $\mathbf{F}(\mathbf{X})$ is the vector that represents the functions of each respective component equation, which are functions of the variables $\mathbf{X}$.

A vector $\mathbf{Y}$ is defined as a displacement $\delta \mathbf{X}$ in the following way: $\mathbf{Y}=\mathbf{X}-\mathbf{X}_{A E P}$, where $\mathbf{X}_{A E P}$ is the vector $\mathbf{X}$ calculated at the AEP. Each component of the function vector $\mathbf{F}(\mathbf{X})$ can be expanded in Taylor series around the AEP. In this series expansion, if the terms of the components of $\mathbf{Y}$ of order two or more are 
neglected, as well as crossed terms among the components of Y, Eq. (43) is said to be linearized around the AEP, and the result is written as

$\frac{\mathrm{d} \mathbf{Y}}{\mathrm{d} t}=\mathbf{A}_{6 \times 6} \mathbf{Y}$,

where $\mathbf{A}_{6 \times 6}$ is a $6 \times 6$ matrix, representing the Jacobian of the function vector $\mathbf{F}(\mathbf{X})$, calculated at the AEP. A perturbation may be added to Eq. (44) and the result is written as

$\frac{\mathrm{d} \mathbf{Y}}{\mathrm{d} t}=\mathbf{A}_{6 \times 6} \mathbf{Y}+\mathbf{p}_{\mathbf{a}}$,

where $\mathbf{p}_{\mathbf{a}}$ is the vector due to the perturbation of the system that comes directly from a in Eq. (1). The perturbation $\mathbf{p}_{\mathbf{a}}$ is assumed to be constant for this kind of solution.

Equation (45) is known as a system of non-homogeneous coupled linear ordinary differential equations of first order with constant coefficients. As said before, this method is expensive; thus, a general solution for general parameters could not be obtained due to the large number of terms contained in the eigenvalues of $\mathbf{A}_{6 \times 6}$. On the other hand, if all the values of the parameters are explicitly defined, the analytical solution $\mathbf{Y}(t)$ of Eq. (45) is obtained through the use of the method of combinations of the eigenvectors of $\mathbf{A}_{6 \times 6}$ plus a particular solution of this equation, taking into account that the initial conditions are given at the AEP $\left(\mathbf{Y}_{0}=\mathbf{0}\right)$.

Linear stability analysis for different explicit possible forms of $\mathbf{f}_{p}$ could be done using $\mathbf{A}_{6 \times 6}$. On the other side, linear stability analysis of AEP in space has already been done in the case of a radial propulsive acceleration [13] or a general propulsive acceleration [19].

\section{Results and analysis}

In this section, the results of the solutions are shown for several more realistic cases, that consider the gravitational perturbations of Jupiter and Venus or just Jupiter. In order to give an estimation of the accuracy of the analytical solutions with the assumptions made by the approximations, numerical simulations of the complete equation of motion [Eq. (3)] are used for comparison purposes.

A spacecraft whose parameters and positions variables are such that the conditions of an AEP are satisfied
Table 1 Values of parameters used in the present paper

$$
\begin{aligned}
& R=1.49597870710^{11} \mathrm{~m}=1 \mathrm{au} \\
& p_{e}=4.5610^{-6} \mathrm{~N} / \mathrm{m}^{2}[29] \\
& \mu_{s}=1.3271244004110^{20} \mathrm{~m}^{3} / \mathrm{s}^{2}[30] \\
& \mu_{e}=\mu_{s} / 328,900.56[30] \\
& \mu_{j}=\mu_{s} / 1047.3486[30] \\
& \mu_{v}=\mu_{s} / 408,523.71[30]
\end{aligned}
$$

Table 2 Parameter values of the artificial equilibrium point

$$
\begin{aligned}
& \frac{A}{m}=12 \mathrm{~m}^{2} / \mathrm{kg} \\
& \gamma_{e}=0.670259715053405 \mathrm{rad} \\
& x_{0}=-1.4915243157291810^{11} \mathrm{~m} \\
& y_{0}=0 \mathrm{~m} \\
& z_{0}=1.059510^{9} \mathrm{~m}
\end{aligned}
$$

near the Lagrangian point $L_{3}$ of the Sun-Earth system is subjected to the perturbation of the gravitational interaction of Jupiter and Venus in the Epoch December 16, 2016. The equation of motion of such spacecraft is described by Eq. (3). An AEP implies that Eq. (4) is satisfied. In order to show a more explicit example, suppose that $\mathbf{f}_{p}$ given in Eq. (4) is the force due to the solar radiation pressure acting over the surface of the solar sail of a spacecraft and it is given by [29]

$\mathbf{f}_{p}=\frac{2 p_{e} A R^{2} \cos ^{2}\left(\gamma_{e}\right)}{r_{s}^{2}} \mathbf{n}$,

where $R$ is the Sun-Earth distance, $p_{e}$ is the solar radiation pressure at a distance $R$ from the Sun, $A$ is the total area of the flat solar sail, $\mathbf{n}$ is the vector normal to the solar sail and $\gamma_{e}$ is the angle between $\mathbf{n}$ and $\mathbf{r}_{\mathbf{s}}$.

The values of the parameters used for all the calculations are given in Table 1 . If the force due to the solar radiation pressure is given by Eq. (46), whose parameters and variables values are given in Table 2, then Eq. (4) is satisfied and the spacecraft is located at an AEP.

At this equilibrium point, Eq. (3) becomes Eq. (5). For the analytical solution 2, the perturbations of Jupiter and Venus are considered constant for the integration time, which is a whole day. This time is very short compared to the orbital periods of Venus and Jupiter, so their positions are considered constants for the analytical solution 2 and also for the linear analytical solution. In the case of the analytical solution 1, the 
Table 3 Values of the positions of Jupiter and Venus [31] in $12 / 16 / 2016$ in the rotating frame of reference

\begin{tabular}{lrr}
\hline Coordinate & \multicolumn{1}{l}{ Jupiter } & \multicolumn{1}{l}{ Venus } \\
\hline$x$ & -166397914578 & 54185387000 \\
$y$ & -721781287016 & 93293222036 \\
$z$ & 16846403798 & -4976020886 \\
$\sqrt{x^{2}+y^{2}+z^{2}}$ & 740905050316 & 108002047323
\end{tabular}

The last line shows the total distance from the Sun. All values are given in meters

perturbation of Venus and Jupiter is calculated at the AEP, but it linearly varies with time. Then, the motion of the spacecraft around the AEP can be determined by the analytical solutions of the equations of motion, which are Eqs. (30-35), (24-29) and (45). These solutions are exact solutions at the AEP, but they are approximations outside this point. The accuracy of each kind of solution is shown, and it is expected to be better when the spacecraft is positioned near the AEP.

For the examples shown here, the spacecraft under the gravitational influence of the Sun, Earth, Venus and Jupiter is positioned at the AEP according to Table 2, with initial velocities given by $v_{x 0}=v_{y 0}=v_{z 0}=$ $0 \mathrm{~m} / \mathrm{s}$ at the time zero. The evolutions of their respective motion are determined and compared by the analytical solution 1 [Eqs. (24-29)], the analytical solution 2 [Eqs. (30-35)], the analytical solution of the linear expansion [solution of Eq. (45)] and the numerical solution integrated via Runge Kutta of fourth order, as shown in the next figures.

\subsection{Case 1: Jupiter and Venus in date of $12 / 16 / 16$}

In this first case, the positions of Venus and Jupiter relative to the frame of reference are calculated for the approximated real data [31] in the date of 12/16/16. These positions at the initial integration time $(t=0)$ are given in Table 3. For the analytical solution 2 and the linear analytical solution, the perturbation is assumed to be constant and its value is calculated at the initial conditions (the AEP and $t=0$ ). For the analytical solution 1, the perturbation is calculated at the AEP at the time $t=0$ and at the AEP at the time $t=t_{f}$, where $t_{f}$ is the final integration time. Thus, two steps are needed: one to calculate the values of $\alpha$ for each component of perturbation and another to calculate the values of the solutions. For the numerical solution, the positions of Jupiter and Venus are updated for each
Table 4 Case 1 -delta function of the components of the position and velocity calculated at the time $t_{f}=86,400 \mathrm{~s}$ for the linear analytical solution, the analytical solution 1 and the analytical solution 2

\begin{tabular}{llll}
\hline & Linear analytical & Analytical sol. 2 & Analytical sol. 1 \\
\hline$\Delta x$ & $-8.0141 \times 10^{-0}$ & $-8.0136 \times 10^{-0}$ & $-3.4466 \times 10^{-0}$ \\
$\Delta y$ & $-1.6452 \times 10^{-0}$ & $-1.6454 \times 10^{-0}$ & $5.2743 \times 10^{-2}$ \\
$\Delta z$ & $0.4496 \times 10^{-0}$ & $0.4501 \times 10^{-0}$ & $0.3955 \times 10^{-0}$ \\
$\Delta v_{x}$ & $-2.3854 \times 10^{-4}$ & $-2.3851 \times 10^{-4}$ & $-7.9772 \times 10^{-5}$ \\
$\Delta v_{y}$ & $-5.6823 \times 10^{-5}$ & $-5.6832 \times 10^{-5}$ & $1.6736 \times 10^{-6}$ \\
$\Delta v_{z}$ & $1.1041 \times 10^{-5}$ & $1.1062 \times 10^{-5}$ & $9.1654 \times 10^{-6}$ \\
\hline
\end{tabular}

The perturbations are given by Jupiter and Venus with their respective positions in the date $12 / 16 / 2016$. The delta function is the difference between the analytical and the numerical solutions. The units are meters for the differences in positions and $\mathrm{m} / \mathrm{s}$ for the differences in velocities

step of the numerical integration, so this solution takes into account the influence of the motions of Jupiter and Venus around the Sun into the perturbation of the system.

There are two sources of errors in the analytical solutions. The first one comes from the displacement of the spacecraft from the AEP, since the analytical solutions are obtained with the assumption that Eq. (4) is always true. The second source of error comes from the acceleration due to the perturbation, since the analytical solution 2 and the linear analytical solution are obtained assuming a constant perturbation, while the analytical solution 1 assumes that this perturbation linearly varies with time. In order to know which solution is better to describe the motion of the spacecraft, the numerical calculations are made considering the perturbation due to the gravitational forces of Jupiter and Venus and updating their values for each integration step for the respective position of the spacecraft, as the solution evolves in time. The updating of the position of Jupiter and Venus in time is obtained through an algorithm given in [31]. Due to this step update, the numerical solution is closer to reality, among these models.

In order to give an idea of the differences in the solutions at the end of the integration time, a delta function is defined as the analytical minus the numerical solution, for each analytical solution and for each of the components of the position and velocity as functions of time, as shown in Eq. (47). Their respective values are shown in Tables 4, 5, 6 and 7, always calculated at the final time of the integration interval $\left(t_{f}=86,400 \mathrm{~s}\right)$. 
Table 5 Case 2-delta function of the components of the position and velocity calculated at the time $t_{f}=86,400 \mathrm{~s}$ for the linear analytical solution, the analytical solution 1 and the analytical solution 2

\begin{tabular}{llll}
\hline & Linear analytical & Analytical sol. 2 & Analytical sol. 1 \\
\hline$\Delta x$ & $-3.3263 \times 10^{-0}$ & $-3.2793 \times 10^{-0}$ & $-3.5030 \times 10^{-0}$ \\
$\Delta y$ & $-1.9969 \times 10^{-0}$ & $-1.9972 \times 10^{-0}$ & $4.7333 \times 10^{-2}$ \\
$\Delta z$ & $0.3896 \times 10^{-0}$ & $0.3890 \times 10^{-0}$ & $0.3994 \times 10^{-0}$ \\
$\Delta v_{x}$ & $-7.4608 \times 10^{-5}$ & $-7.2433 \times 10^{-5}$ & $-7.9996 \times 10^{-5}$ \\
$\Delta v_{y}$ & $-6.9422 \times 10^{-5}$ & $-6.9441 \times 10^{-5}$ & $1.5703 \times 10^{-6}$ \\
$\Delta v_{z}$ & $8.8993 \times 10^{-6}$ & $8.8704 \times 10^{-6}$ & $9.2303 \times 10^{-6}$ \\
\hline
\end{tabular}

The perturbations are given by Jupiter and Venus initially positioned at $t=0$ in $(-740905050316,0,0)$ and $(-108002047323,0,0)$, respectively. The delta function is the difference between the analytical and the numerical solutions. The units are meters for the differences in positions and $\mathrm{m} / \mathrm{s}$ for the differences in velocities

Table 6 Case 3-delta function of the components of the position and velocity calculated at the time $t_{f}=86,400 \mathrm{~s}$ for the linear analytical solution, the analytical solution 1 and the analytical solution 2

\begin{tabular}{llll}
\hline & Linear analytical & Analytical sol. 2 & Analytical sol. 1 \\
\hline$\Delta x$ & $-3.4025 \times 10^{-0}$ & $-3.4481 \times 10^{-0}$ & $-3.4800 \times 10^{-0}$ \\
$\Delta y$ & $2.5986 \times 10^{-0}$ & $2.5989 \times 10^{-0}$ & $3.8396 \times 10^{-2}$ \\
$\Delta z$ & $0.3948 \times 10^{-0}$ & $0.3949 \times 10^{-0}$ & $0.3949 \times 10^{-0}$ \\
$\Delta v_{x}$ & $-7.8135 \times 10^{-5}$ & $-8.0242 \times 10^{-5}$ & $-8.1607 \times 10^{-5}$ \\
$\Delta v_{y}$ & $9.0268 \times 10^{-5}$ & $9.0286 \times 10^{-5}$ & $1.3852 \times 10^{-6}$ \\
$\Delta v_{z}$ & $9.1397 \times 10^{-6}$ & $9.1438 \times 10^{-6}$ & $9.1433 \times 10^{-6}$
\end{tabular}

The perturbations are given by Jupiter and Venus initially positioned at $t=0$ in $(740905050316,0,0)$ and (108002047323, 0, 0), respectively. The delta function is the difference between the analytical and the numerical solutions. The units are meters for the differences in positions and $\mathrm{m} / \mathrm{s}$ for the differences in velocities

$$
\begin{aligned}
& \Delta x=\left.\left(x-x_{0}\right)\right|_{\text {analytical }}-\left.\left(x-x_{0}\right)\right|_{\text {numerical }} \\
& \Delta v_{x}=\left.v_{x}\right|_{\text {analytical }}-\left.v_{x}\right|_{\text {numerical }} \\
& \Delta y=\left.\left(y-y_{0}\right)\right|_{\text {analytical }}-\left.\left(y-y_{0}\right)\right|_{\text {numerical }} \\
& \Delta v_{y}=\left.v_{y}\right|_{\text {analytical }}-\left.v_{y}\right|_{\text {numerical }} \\
& \Delta z=\left.\left(z-z_{0}\right)\right|_{\text {analytical }}-\left.\left(z-z_{0}\right)\right|_{\text {numerical }} \\
& \Delta v_{z}=\left.v_{z}\right|_{\text {analytical }}-\left.v_{z}\right|_{\text {numerical }} .
\end{aligned}
$$

The differences between the values of the positions as functions of $t$ and their values in the initial conditions are shown in Fig. 2 with the respective values of $\Delta$. The values of the components of the velocity
Table 7 Case 4-delta function of the components of the position and velocity calculated at the time $t_{f}=86,400 \mathrm{~s}$ for the linear analytical solution, the analytical solution 1 and the analytical solution 2

\begin{tabular}{llll}
\hline & Linear analytical & Analytical sol. 2 & Analytical sol. 1 \\
\hline$\Delta x$ & $-8.0470 \times 10^{-0}$ & $-8.0449 \times 10^{-0}$ & $3.8823 \times 10^{-0}$ \\
$\Delta y$ & $-1.6990 \times 10^{-0}$ & $-1.6992 \times 10^{-0}$ & $3.2995 \times 10^{-0}$ \\
$\Delta z$ & $0.4558 \times 10^{-0}$ & $0.4563 \times 10^{-0}$ & $0.1840 \times 10^{-0}$ \\
$\Delta v_{x}$ & $-2.3969 \times 10^{-4}$ & $-2.3858 \times 10^{-4}$ & $1.7505 \times 10^{-4}$ \\
$\Delta v_{y}$ & $-5.8687 \times 10^{-5}$ & $-5.8697 \times 10^{-5}$ & $1.1368 \times 10^{-4}$ \\
$\Delta v_{z}$ & $1.1255 \times 10^{-5}$ & $1.1277 \times 10^{-5}$ & $1.8235 \times 10^{-6}$ \\
\hline
\end{tabular}

The perturbation is given only by Jupiter with its respective position in the date $12 / 16 / 2016$. The delta function is the difference between the analytical and the numerical solutions. The units are meters for the differences in positions and $\mathrm{m} / \mathrm{s}$ for the differences in velocities

are shown in Fig. 3 also with the respective values of $\Delta$. The total components of the perturbation a at the AEP are $a_{x}=9.110^{-11} \mathrm{~m} / \mathrm{s}^{2}, a_{y}=-2.410^{-7} \mathrm{~m} / \mathrm{s}^{2}$ and $a_{z}=5.110^{-9} \mathrm{~m} / \mathrm{s}^{2}$. The large differences among these components explain why the motion is larger in the $y$ axis, as shown in Fig. 2. It can be noted, from this figure, that the analytical and numerical solutions apparently diverge faster for $x$. They are almost identical for $y$, and they are slightly divergent for $z$. It can also be noted that, as expected, the solutions are almost coincident for low values of $t$, starting from a perfect match at $t=0$. The range of the validity of the analytical solutions depends on the accuracy required. For the example shown in Fig. 2, the major difference is in the $x$ component. In the worst case of the $x$-axis, the errors (differences between analytical and numerical solutions) are below $0.2 \mathrm{~m}$ in position and $10^{-4} \mathrm{~m} / \mathrm{s}$ in velocity in the first $20,000 \mathrm{~s}$, which is more than $5 \mathrm{~h}$. This means a result good enough to consider that the analytical equations are useful, even if Eq. (4) is true only in a single point, which is the starting point of the motion. Figure 2 also shows that the analytical solution 2 and the linear solution are almost coincident with each other and the analytical solution 1 approximates the numerical solution more than the other two options. The solution is better when the curve that represents $\Delta$ shown in the right side of Figs. 2 and 3is closer to zero. The comparisons among the curves in these figures also indicates that, for the $x$ coordinate, approximately almost half of the error of the analytical solution 2 and the linear solution is due to the assump- 

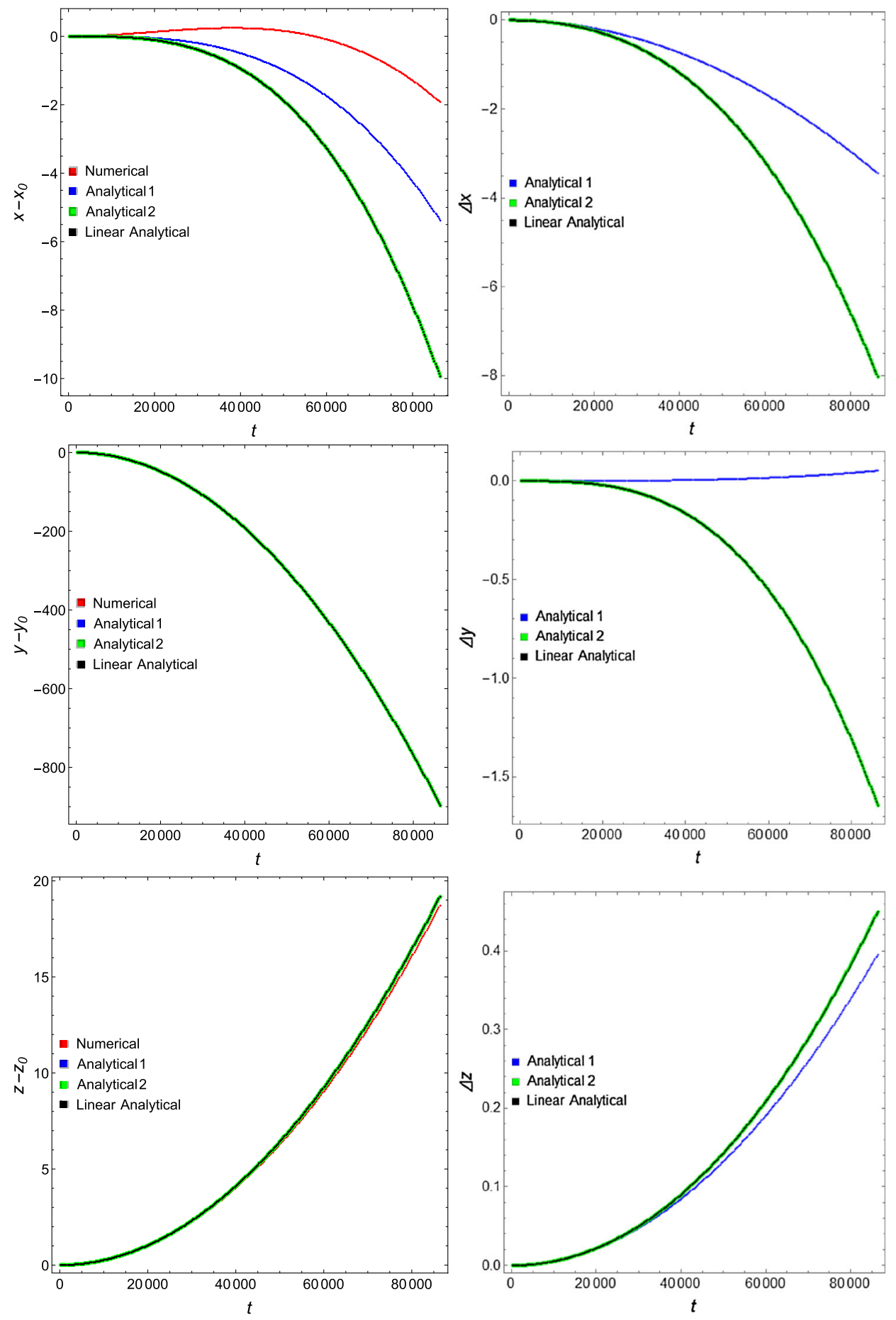

Fig. 2 Case 1-components of the differences between the position and the initial position are shown in the three figures of the left side as functions of time evaluated numerically (red), analytically via solution 1 (blue), analytically via solution 2 (green) and analytically via linearized equations of motion (black). The functions $\Delta$ defined in Eq. (47) are shown in the figures of the

right side for the components of the position. At the time $t=0$, the spacecraft is located at the AEP given in Table 2 with velocities $v_{x 0}=v_{y 0}=v_{z 0}=0 \mathrm{~m} / \mathrm{s}$. The motion of the perturbing planets Jupiter and Venus around the Sun is calculated using [31]. Note that overlays of the curves may arise hiding firstly the red one in the figures of the left side. (Color figure online) 

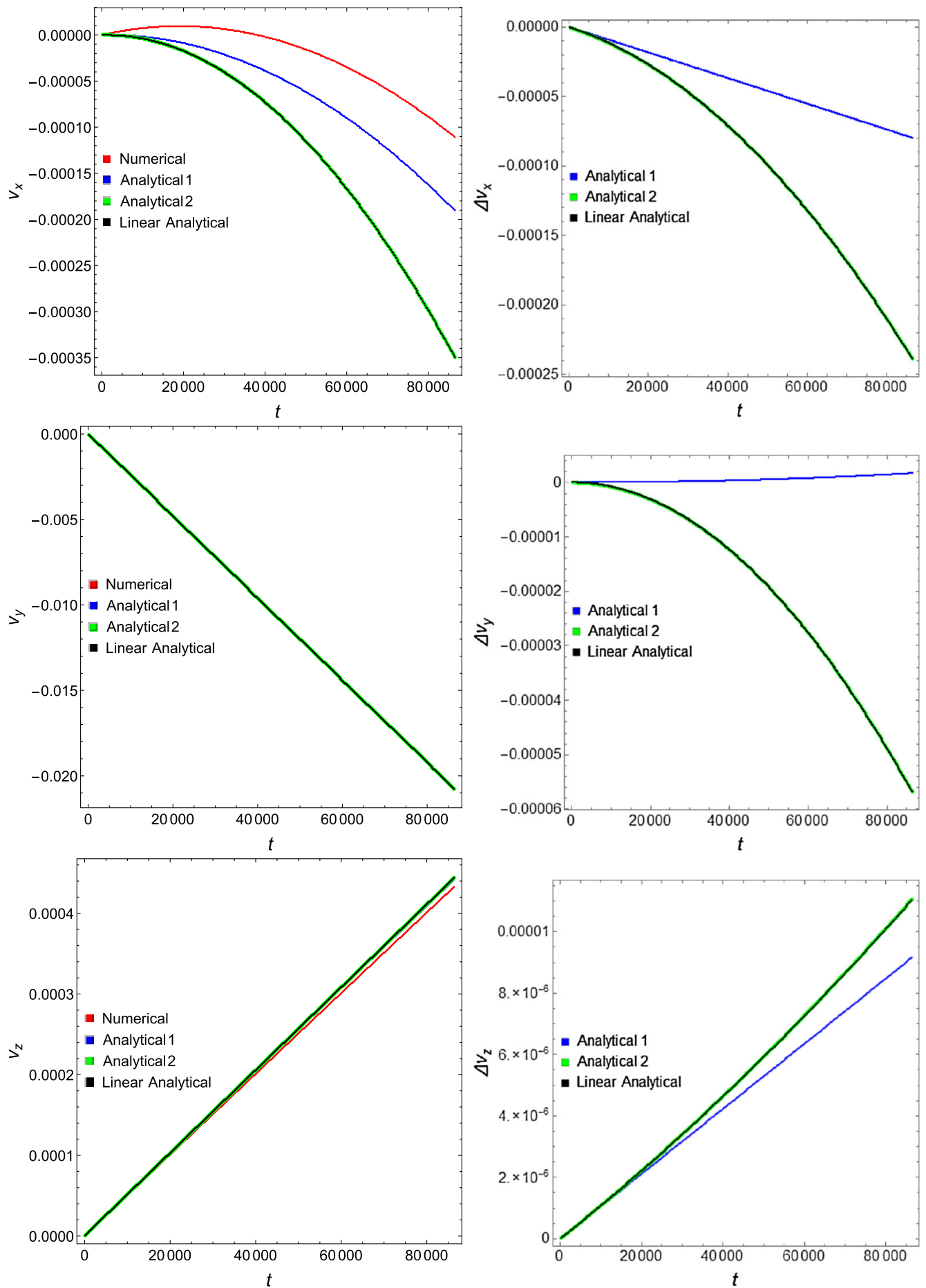

Fig. 3 Case 1 -components of the velocity are shown in the three figures of the left side as functions of time evaluated numerically (red), analytically via solution 1 (blue), analytically via solution 2 (green) and analytically via linearized equations of motion (black). The functions $\Delta$ defined in Eq. (47) are shown in the figures of the right side for the components of the velocity.

At the time $t=0$, the spacecraft is located at the AEP given in Table 2 with velocities $v_{x 0}=v_{y 0}=v_{z 0}=0 \mathrm{~m} / \mathrm{s}$. The motion of the perturbing planets Jupiter and Venus around the Sun is calculated using [31]. Note that overlays of the curves may arise hiding firstly the red one in the figures of the left side. (Color figure online) 

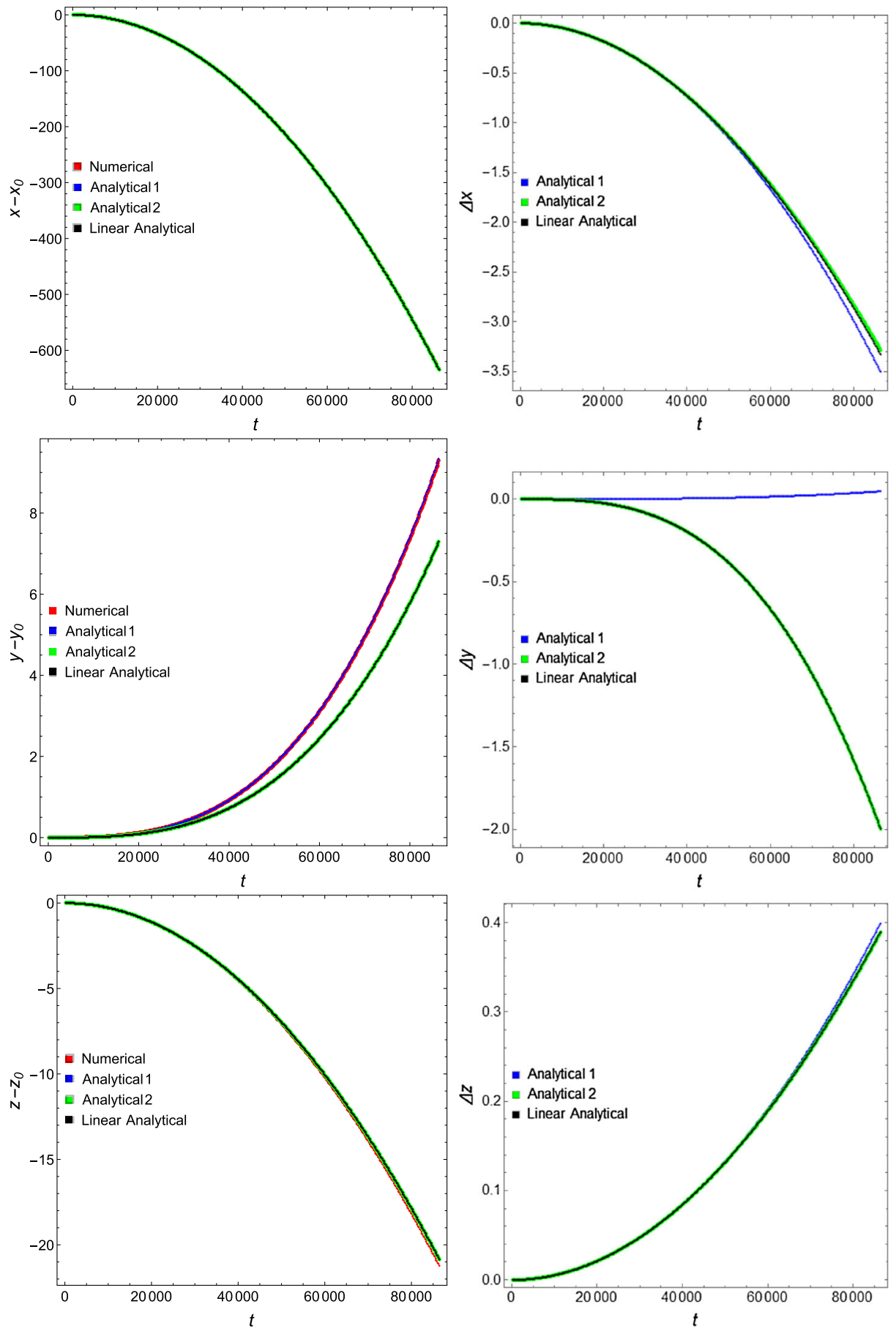

Fig. 4 Case 2-components of the differences between the position and the initial position are shown in the three figures of the left side as functions of time evaluated numerically (red), analytically via solution 1 (blue), analytically via solution 2 (green) and analytically via linearized equations of motion (black). The functions $\Delta$ defined in Eq. (47) are shown in the figures of the right side for the components of the position. At the time

$t=0$, the spacecraft is located at the AEP given in Table 2 with velocities $v_{x 0}=v_{y 0}=v_{z 0}=0 \mathrm{~m} / \mathrm{s}$. Jupiter and Venus are initially positioned at the nearest position to the spacecraft at $(-740905050316,0,0)$ and $(-108002047323,0,0)$, respectively. Note that overlays of the curves may arise hiding firstly the red one in the figures of the left side. (Color figure online) 

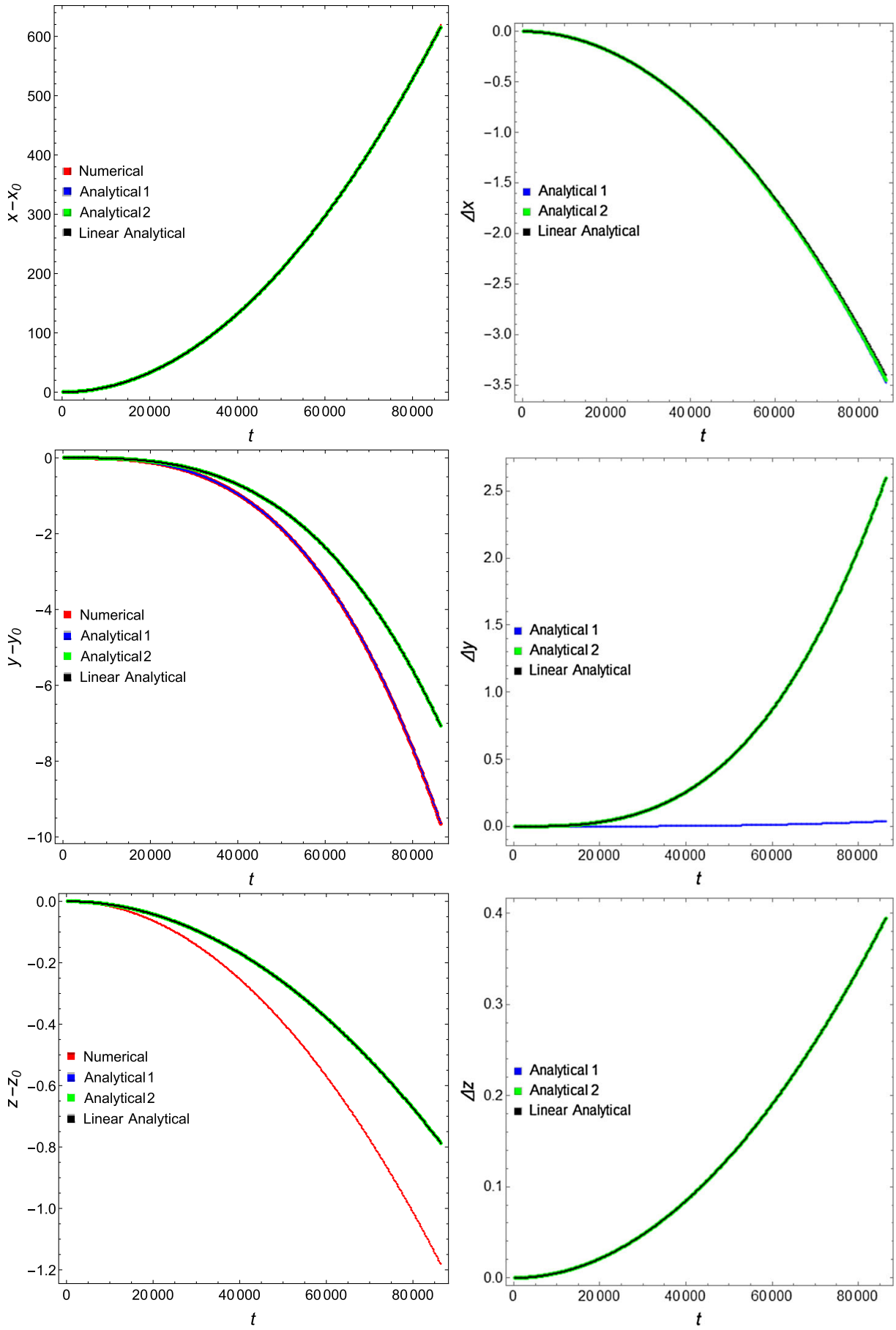

Fig. 5 Case 3-components of the differences between the position and the initial position are shown in the three figures of the left side as functions of time evaluated numerically (red), analytically via solution 1 (blue), analytically via solution 2 (green) and analytically via linearized equations of motion (black). The functions $\Delta$ defined in Eq. (47) are shown in the figures of the right side for the components of the position. At the time

$t=0$, the spacecraft is located at the AEP given in Table 2 with velocities $v_{x 0}=v_{y 0}=v_{z 0}=0 \mathrm{~m} / \mathrm{s}$. The position of Jupiter and Venus at $t=0$ is $(x, y, z)=(740905050316,0,0)$ and $(x, y, z)=(108002047323,0,0)$, respectively. Note that overlays of the curves may arise hiding firstly the red one in the figures of the left side. (Color figure online) 

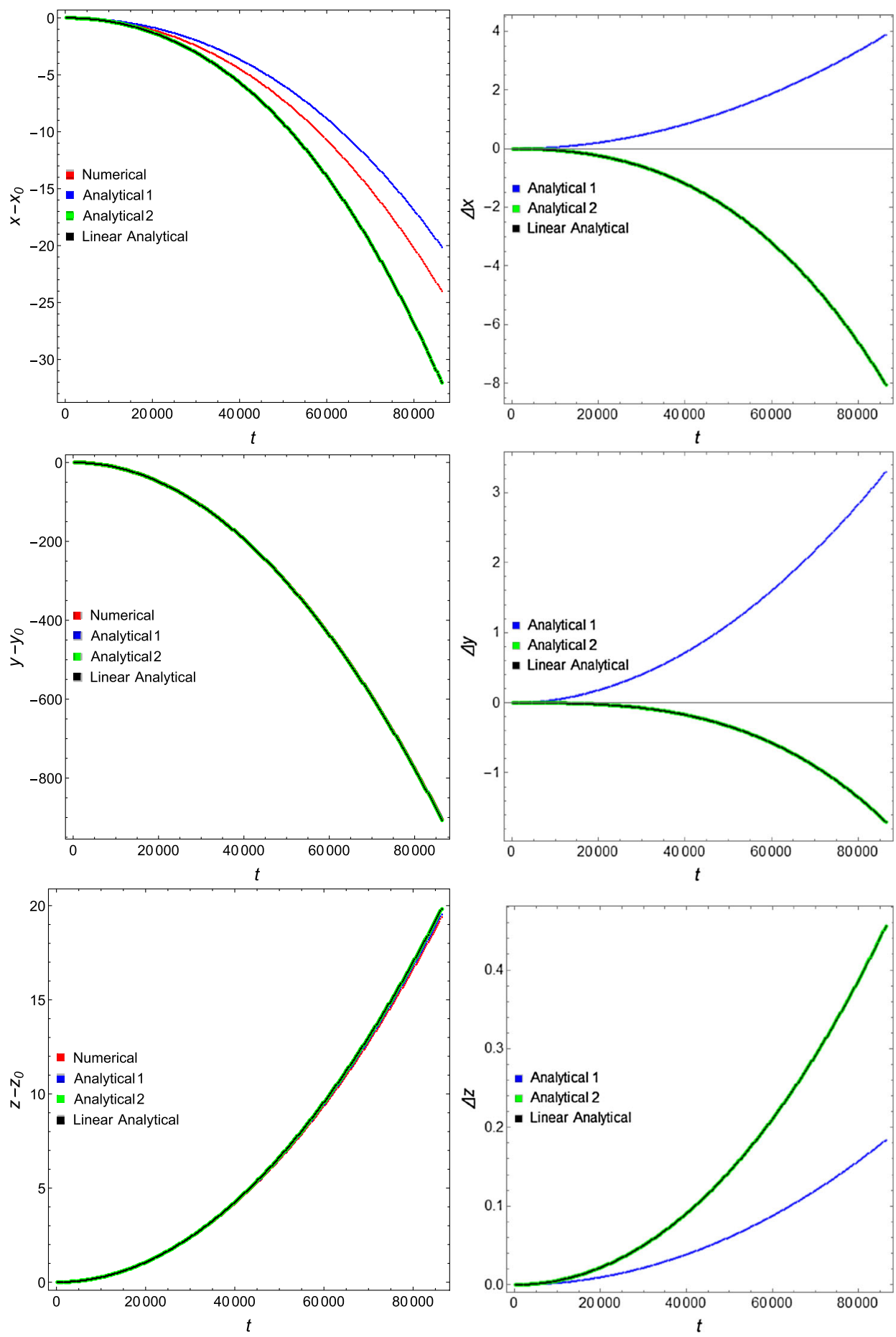

Fig. 6 Case 4-components of the differences between the position and the initial position are shown in the three figures of the left side as functions of time evaluated numerically (red), analytically via solution 1 (blue), analytically via solution 2 (green) and analytically via linearized equations of motion (black). The functions $\Delta$ defined in Eq. (47) are shown in the figures of the

right side for the components of the position. At the time $t=0$, the spacecraft is located at the AEP given in Table 2 with velocities $v_{x 0}=v_{y 0}=v_{z 0}=0 \mathrm{~m} / \mathrm{s}$. The perturbation is given by only the gravitational force of Jupiter calculated using [31]. Note that overlays of the curves may arise hiding firstly the red one in the figures of the left side. (Color figure online) 
tion that the perturbations from Venus and Jupiter over the spacecraft are constants.

Table 4 indicates that the delta of the analytical solution 1 is the function that tends to get closer to zero, which means that this is the solution closer to the numerical solution, for the given perturbation.

3.2 Case 2: Jupiter and Venus at $\left(-R_{J}, 0,0\right)$ and $\left(-R_{V}, 0,0\right)$, respectively

In order to continue the analysis of the influence of the perturbations over the differences between the four kinds of analytical and numerical solutions for more situations, Jupiter is set to be in the nearest position of the spacecraft (located near $L_{3}$ ) in the initial position in the frame of reference $(x, y, z)=\left(-R_{J}, 0,0\right)$. Venus is also set to be in the nearest position of the spacecraft, so its positions is $(x, y, z)=\left(-R_{V}, 0,0\right)$, where $R_{J}$ and $R_{V}$ are the distance of these planets from the Sun at $12 / 16 / 16$. The values of $R_{J}$ and $R_{V}$ are given by $\sqrt{x^{2}+y^{2}+z^{2}}$ in Table 3 , for each planet. Their motion is assumed to be circular around the Sun with the constant angular velocity given by $\sqrt{\mu_{s} / R_{J}^{3}}-w$ for Jupiter and $\sqrt{\mu_{s} / R_{V}^{3}}-w$ for Venus during the interval of integration time, which is a day $\left(t_{f}=86,400 \mathrm{~s}\right)$. The results are shown in Fig. 4 and Table 5 as the difference in the positions as functions of time and the $\Delta$ functions.

\subsection{Case 3: Jupiter and Venus at $\left(R_{J}, 0,0\right)$ and $\left(R_{V}, 0,0\right)$, respectively}

The same analysis can be made by changing the position of Jupiter in the frame of reference to $(x, y, z)=$ $\left(R_{J}, 0,0\right)=(740905050316,0,0)$ and Venus to $(x, y, z)=\left(R_{V}, 0,0\right)=(108002047323,0,0)$. Their motions around the Sun are also assumed to be circular with constant angular velocity during the integration time. The results are shown in Fig. 5 and Table 6 as the difference in the positions as functions of time and the $\Delta$ functions.

\subsection{Case 4: Jupiter only}

In order to analyze the behavior of the analytical solutions for a gravitational perturbation caused by only a single planet, the same results obtained before with the perturbations coming from Jupiter and Venus (Figs. 2, 3 and Table 4) are shown neglecting the Venus gravitational influence over the spacecraft. This means that the perturbation is caused only by the gravitational influence of Jupiter. The results are shown in Fig. 6 and in Table 7.

\section{Conclusions}

Three kinds of solutions were developed analytically with the objective to describe the motion of a spacecraft around an AEP. The three solutions are analytical 1 [Eqs. (24-29)], analytical 2 [Eqs. (30-35)] and the linear analytical [given by the solution of Eq. (45)]. All of them can describe the motion around the AEP.

The numerical solution is the closest to the reality among the solutions, because it does not use approximations for the condition given by Eq. 4 outside the AEP and the values of the perturbations are updated in each small step of the integration time. This means that the definitions given by Eq. (47) for the delta functions allow them to describe the error associated with each one of the analytical solutions found in this research. Of course, this error decreases with the decreasing time and it tends to zero for shorter integration times. Thus, all the solutions are able to describe the motion of the spacecraft around the AEP with some degree of accuracy, which also means that an error is associated with each one of the analytical solutions outside the AEP. In this research, these errors are estimated for some cases, allowing the reader to analyze the behavior of the error when the system is evolved for a day.

Tables 4, 5, 6 and 7 show that the maximum absolute values of the delta function are of the order of $1 \mathrm{~m}$ for the components of the position and of the order of $10^{-4} \mathrm{~m} / \mathrm{s}$ for the components of the velocity, if the systems are propagated for a whole day. This means that a day of integration time can associate any of the solutions obtained in this research with errors that could fit in the design of some spacecraft missions.

The analytical solution 2 and the linear analytical solution are always practically coincident for all the positions of Jupiter and Venus used in Sect. 3, as shown in Figs. 2, 3, 4, 5 and 6 and Tables 4, 5, 6 and 7. This is an expected result, since both of them consider the perturbation as a constant of motion. On the other hand, both solutions are obtained through two completely different 
methods. Linear analytical solutions require very complex steps, in which the final solution $\mathbf{Y}(t)$ is only found if the parameters are explicit, while analytical solution 2 takes the advantage of a general solution obtained in a closed form for all values of the parameters.

Tables 4, 5, 6 and 7 show that the absolute values of the delta function for analytical solution 1 are the one among all analytical solutions that tends to be closer to zero, but there are some exceptions. For the first case, shown in Sect. 3.1, the absolute values of the delta functions for analytical solution 1 are smaller than both linear analytical and analytical solution 2 for every position and velocity components. For the second case, shown in Sect. 3.2, Table 5 shows that the absolute values of the delta function are slightly larger for the analytical solution 1 than others, both for $x$ and $z$ axis components, but all these errors are small if compared to the respective axis displacement. The errors are about $0.5 \%$ of the displacement for $x$ and about $2 \%$ for $z$ position components. Otherwise, an analysis in the $y$ position components shows that the error is larger for the analytical solution 2 and the linear analytical solution. These errors are larger if compared to the displacement, about $25 \%$ of the displacement for the analytical solution 2 and for the linear analytical solution. On the other hand, the comparative error for the analytical solution 1 shows that the error is only about $0.05 \%$ of the displacement in $y$ position components. This fact indicates that, for this case, analytical solution 1 is the best choice. For the third case, shown in Sect. 3.3, an analysis of Table 6 shows quite similar results compared to the second case, except that all the solutions show significant errors for the $z$ position component. The error is about $33 \%$ in the displacement, but the $z$ displacement is much smaller for this case than for the other two situations shown before. For the fourth case, shown in Sect. 3.4, Table 7 shows that the absolute values of the delta function for analytical solution 88 is smaller for $x$ and $z$ components position and it is larger for $y$ component position. For the linear analytical solution and analytical solution 2 , the errors are: about $26 \%$ of the displacement for $x$ coordinate, about $0.17 \%$ of the displacement for $y$ coordinate and about $2.3 \%$ of the displacement for $z$ coordinate. This same table shows that the errors for the analytical solution 88 are about $12.6 \%$ of the displacement for $x$ coordinate, about $0.33 \%$ of the displacement for $y$ coordinate and about $0.9 \%$ of the displacement for $z$ coordinate. Again, when the relative error is large, the analytical solution 1 is much better than the other two analytical solutions. All these results can be also analyzed through Figs. 2, 3, 4, 5 and 6, because they show the differences between the analytical solutions for all the interval of the integration, not only in the final time. Thus, the conclusion is that the errors of the analytical solution 1 tend to be smaller than the errors of the analytical solution 2 , which in turn are quite similar to the errors of the linear analytical solution.

If Eq. (4) is satisfied along all the path, then analytical solutions 1 and 2 are exact ones for their respective given perturbations. Moreover, all kinds of analytical solutions presented in this paper can be considered as approximated ones in more realistic cases, where Eq. (4) is satisfied only in the initial conditions of the motion and the perturbations are approximated as constants or linearly dependent on time. In these more realistic cases, the numerical solution is closer to reality. On the other hand, for the numerical calculations, more than a million operations are needed in order to reach the results, while the analytical calculations could be made for any time with less than a thousand operations. An analytical solution can be useful for many purposes, including to lower time computation costs or analytical calculations of station-keeping costs, trajectories.

Acknowledgements The authors acknowledge financial support from CAPES - Coordination for the Improvement of Higher Education Personnel; from CNPQ - National Council for Scientific and Technological Development, Grants 305834/20134, 406841/2016-0 and 301338/2016-7; and from FAPESP-São Paulo Research Foundation, Grants 2016/14665-2, 2016/245610, 2014/22293-2 and 2014/22295-5.

\section{References}

1. Williams, K.E.: Overcoming Genesis mission design challenges. Acta Astronaut. 52, 281-287 (2003)

2. Tantardini, M., Fantino, E., Ren, Y., Pergola, P., Gomez, G., Masdemont, J.: Spacecraft trajectories to the $L_{3}$ point of the Sun-Earth three-body problem. Celest. Mech. Dyn. Astron. 108(3), 215-232 (2010)

3. Jiang, Y., et al.: Order and chaos near equilibrium points in the potential of rotating highly irregular-shaped celestial bodies. Nonlinear Dyn. 83, 231 (2016)

4. Gomez, G., Jorba, A., Masdemont, J., Simo, C.: Study of the transfer from the Earth to a halo orbit around the equilibrium point $L_{1}$. Celest. Mech. Dyn. Astron. 56(4), 541-562 (1993)

5. Jorba, A., Masdemont, J.: Dynamics in the centre manifold of the collinear points of the restricted three body problem. Physica D 132, 189-213 (1999) 
6. Gomez, G., Masdemont, J., Simo, C.: Quasi-halo orbits associated with libration points. J. Astronaut. Sci. 46, 135-176 (1998)

7. Koon, W.S., Lo, M.W., Marsden, J.E., Ross, S.D.: Heteroclinic connections between periodic orbits and resonance transition in celestial mechanics. Chaos 10(2), 427-469 (2000)

8. Llibre, J., Martinez, R., Simo, C.: Transversality of the invariant manifolds associated to the Lyapunov family of periodic orbits near $L_{2}$ in the restricted three-body problem. J. Differ. Equ. 48, 104-156 (1985)

9. Barrabes, E., Olle, M.: Invariant manifolds of $L_{3}$ and horseshoe motion in the restricted three-body problem. Nonlinearity 19, 2065-2090 (2006)

10. Prado, A.F.B.A., Broucke, R.A.: Transfer orbits in the restricted problem. J. Guid. Control Dyn. 18(3), 593-598 (1995)

11. Hou, X., Tang, J., Liu, L.: Transfer to the Collinear Libration Point $L_{3}$ in the Sun-Earth + Moon System. Nasa Technical Report. 20080012700 (2007)

12. McInnes, C.R., McDonald, A.J.C., Simmons, J.F.L., MacDonald, E.W.: Solar sail parking in restricted three-body systems. J. Guid. Control Dyn. 17(2), 399-406 (1994)

13. Aliasi, G., Mengali, G., Quarta, A.A.: Artificial equilibrium points for a generalized sail in the circular restricted threebody problem. Celest. Mech. Dyn. Astron. 110(4), 343-368 (2011)

14. Ammar, M.K.: The effect of solar radiation pressure on the Lagrangian points in the elliptic restricted three-body problem. Astrophys. Space Sci. 313, 393 (2008)

15. Tsiolkovsky, K.E.: Extension of man into outer space. In: Proceedings of Symposium Jet Propulsion, vol. 2. United Scientific and Technical Presses (1936)

16. Tsander, K.: From a Scientific Heritage. NASA Technical Translation No. TTf-541, NASA, Washington (1967)

17. Forward, R.L.: Statite-a spacecraft that does not orbit. J. Spacecr. Rockets 28(5), 606-611 (1991)

18. Aliasi, G., Mengali, G., Quarta, A.A.: Artificial equilibrium points for a generalized sail in the elliptic restricted three-body problem. Celest. Mech. Dyn. Astron. 114, 181200(2012)
19. Bombardelli, C., Pelaez, J.: On the stability of artificial equilibrium points in the circular restricted three-body problem. Celest. Mech. Dyn. Astron. 109(1), 1326 (2011)

20. Ranjana, K., Kumar, V.: On the artificial equilibrium points in a generalized restricted problem of three bodies. Int. J. Astron. Astrophys. 3, 508-516 (2013)

21. Salazar, F.J.T., McInnes, C.R., Winter, O.C.: Intervening in Earth's climate system through space-based solar reflectors. Adv. Space Res. 58, 17-29 (2016)

22. McInnes, C.R.: Space-based geoengineering: challenges and requirements. Proc. Inst. Mech. Eng. C J. Mech. Eng. Sci. 224(3), 571-580 (2010)

23. Morimoto, M.Y., Yamakawa, H., Uesugi, K.: Artificial equilibrium points in the low-thrust restricted three-body problem. J. Guid. Control Dyn. 30(5), 1563-1567 (2007)

24. Li, J., Post, M.A., Vukovich, G.: Orbit and attitude stability criteria of solar sail on the displaced orbit. AAS 15, 604 (2015)

25. Janhunen, P., Sandroos, A.: Simulation study of solar wind push on a charged wire: basis of solar wind electric sail propulsion. Ann. Geophys. 25, 755-767 (2007)

26. Mengali, G., Quarta, A.A.: Non-Keplerian orbits for electric sails. Celest. Mech. Dyn. Astron. 105, 179195 (2009)

27. Symon, K.R.: Mechanics, 2nd edn. Campus Ltda, Rio de Janeiro (1986)

28. Boyce, W.E., DiPrima, R.C.: Elementary Differential Equations and Boundary Value Problems, 7th edn. Wiley, New York (2001)

29. McInnes, Colin R.: Solar Sailing Technology, Dynamics and Mission Applications. Springer, Berlin (2004)

30. Luzum, B., et al.: The IAU 2009 system of astronomical constants: the report of the IAU working group on numerical standards for Fundamental Astronomy. Celest. Mech. Dyn. Astron. 110, 293 (2011)

31. http://ssd.jpl.nasa.gov/?planets. Accessed 16 Dec 2016 